\title{
Deep convolutional neural network-based classification of cancer cells on cytological pleural effusion images
}

\author{
Xiaofeng $\mathrm{Xie}^{1}$, Chi-Cheng $\mathrm{Fu}^{2}$, Lei $\mathrm{Lv}^{2}$, Qiuyi Ye ${ }^{2}$, Yue $\mathrm{Yu}^{2}$, Qu Fang ${ }^{2}$, Liping Zhang ${ }^{1}$ Likun Hou ${ }^{1}$ and Chunyan Wu (D) ${ }^{1 凶}$
}

(c) The Author(s) 2022

Lung cancer is one of the leading causes of cancer-related death worldwide. Cytology plays an important role in the initial evaluation and diagnosis of patients with lung cancer. However, due to the subjectivity of cytopathologists and the regiondependent diagnostic levels, the low consistency of liquid-based cytological diagnosis results in certain proportions of misdiagnoses and missed diagnoses. In this study, we performed a weakly supervised deep learning method for the classification of benign and malignant cells in lung cytological images through a deep convolutional neural network (DCNN). A total of 404 cases of lung cancer cells in effusion cytology specimens from Shanghai Pulmonary Hospital were investigated, in which 266, 78, and 60 cases were used as the training, validation and test sets, respectively. The proposed method was evaluated on 60 whole-slide images (WSIs) of lung cancer pleural effusion specimens. This study showed that the method had an accuracy, sensitivity, and specificity respectively of $91.67 \%, 87.50 \%$ and $94.44 \%$ in classifying malignant and benign lesions (or normal). The area under the receiver operating characteristic (ROC) curve (AUC) was 0.9526 (95\% confidence interval (Cl): 0.9019-9.9909). In contrast, the average accuracies of senior and junior cytopathologists were $98.34 \%$ and $83.34 \%$, respectively. The proposed deep learning method will be useful and may assist pathologists with different levels of experience in the diagnosis of cancer cells on cytological pleural effusion images in the future.

Modern Pathology (2022) 35:609-614; https://doi.org/10.1038/s41379-021-00987-4

\section{INTRODUCTION}

Lung cancer is one of the leading causes of cancer-related death worldwide ${ }^{1}$. With the improvement of computed tomography screening technology, small lesions could be detected, making it possible for more patients in the early stage to be cured. However, there are still many patients who are already in a late stage when diagnosed and have missed the best surgical treatment period ${ }^{2,3}$. How to precisely treat this group of patients and improve their disease-free survival and overall survival is key to the diagnosis and treatment of advanced lung cancer ${ }^{4}$.

Patients with advanced lung cancer are often in poor physical condition and can hardly tolerate invasive examinations. This is because bleeding or other complications could easily occur during the procedure due to the large tumor loads. Therefore, it is of great benefit to patients to diagnose, classify (e.g. to distinguish between small cell and non-small cell carcinomas) and stage tumors through minimally invasive approaches, and this remains a popular research direction towards precision therapy ${ }^{5,6}$. Whilst histological biopsy and liquid-based cytological test (LCT) are both minimally invasive approaches for diagnosing lung cancer, the latter is even less invasive, since the specimens are harvested through sputum, pleural effusion, endobronchial ultrasoundguided transbronchial needle aspiration, bronchoscopy brush examination, bronchoalveolar lavage fluid, etc. ${ }^{7,8}$

Pleural effusion commonly exhibits in advanced lung cancer patients, especially in those with lung adenocarcinoma, for whom LCT is often the first-line diagnostic test to determine the stage of tumor and to obtain a large number of tumor cells for further molecular examinations ${ }^{9-11}$. Although many patients with advanced lung cancer are diagnosed cytologically with pleural effusion aspiration in our daily practice, differentiating cancer cells from reactive mesothelial cells remains a challenging problem for pleural effusion cytological diagnosis ${ }^{12}$. Cell blocks and immunohistochemistry $(\mathrm{IHC})$ are not suitable for every cytological specimen because of the limited amount of tissue; thus, cytology slides are often the only sample available for diagnosis. In these cases, cytological morphology is important. However, subjective observation of cytological morphology may result in low interobserver consistency, especially in confusing cases, even for senior pathologists ${ }^{13}$. Improving the discrimination of tumor cells and other cells can provide support for decision making in clinical practice and greatly reduce both physical and economic burdens ${ }^{14-16}$.

Artificial intelligence ( $\mathrm{Al}$ ) has been widely used in the field of modern medicine ${ }^{17,18}$ and can help pathologists make more accurate diagnoses ${ }^{19-22}$. Using a deep convolutional neural network (DCNN), Al can be used to establish a systematic method to evaluate cells and obtain a final result ${ }^{23-26}$. We focus on the applications of $\mathrm{Al}$ to cytology as the latter not only plays an important role in pathology but also has the potential to resolve many clinical problems ${ }^{27}$. The conventional predictive Al models used in decision support systems for medical image analysis rely

\footnotetext{
${ }^{1}$ Department of Pathology, Tongji University Affiliated Shanghai Pulmonary Hospital, Shanghai, China. ${ }^{2}$ Shanghai Aitrox Technology Corporation Limited, Shanghai, China. 凶email: wuchunyan581@sina.com
}

Received: 1 April 2021 Revised: 20 November 2021 Accepted: 22 November 2021

Published online: 10 January 2022 


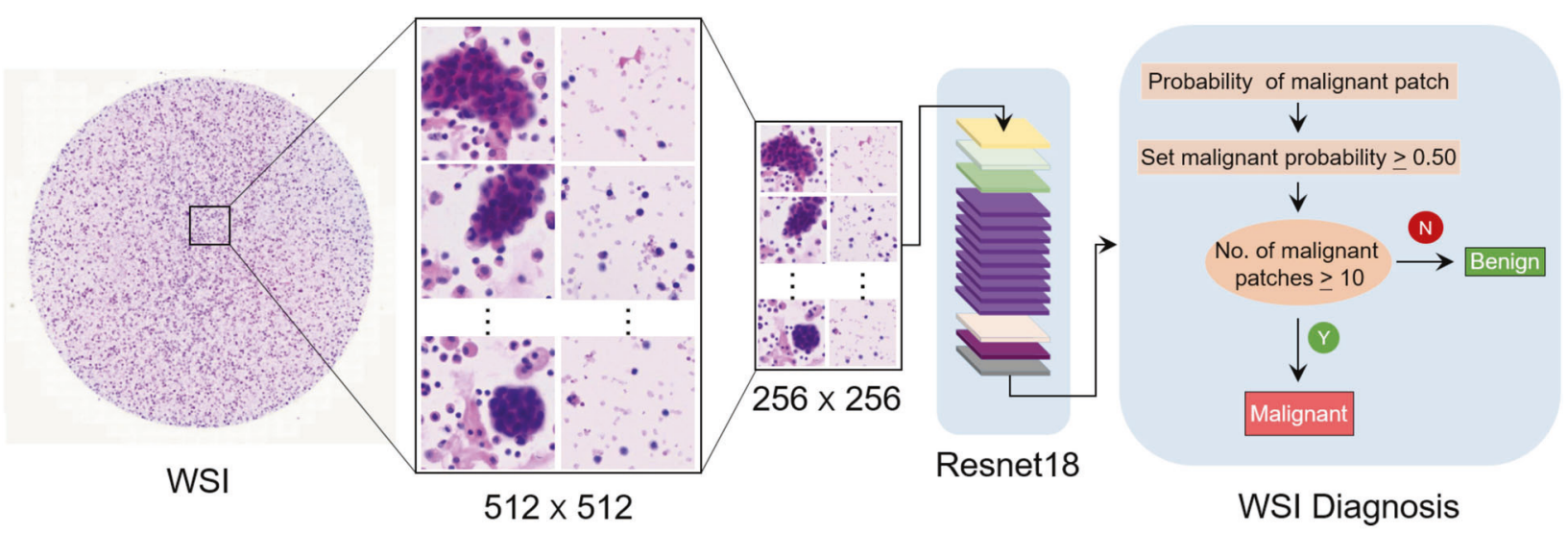

Fig. 1 Overview of the proposed deep learning framework presented in this study. WSIs of lung cancer pleural effusion specimens were cropped into small patches and classified as benign or malignant lesions based on a Resnet 18 deep convolutional neural network. WSI, whole-slide image.

on annotations and manually engineered feature extraction, which is time-consuming and requires the advanced skills of cytopathologists or experts 28,29 . However, the weakly supervised deep learning algorithm could solve the problem of mass annotation, thus not requiring any annotation but a label for samples to be used for the training and validation of the model ${ }^{30-32}$.

In this study, we performed a weakly supervised deep learning method (namely "Aitrox Al model") for the classification of benign and malignant cases based on lung cytological images at the whole-slide image (WSI) level. To investigate the diagnostic performance of the Aitrox Al model, it was compared against that of junior cytopathologists (resident pathologists who studied thoracic cytology for less than 1 year) and senior cytopathologists (attending pathologists who independently handled cytological reports for more than 3 years) with the patient pathological reports used as the gold standard.

\section{MATERIALS AND METHODS}

This study was approved by the Institutional Ethics Committee of Shanghai Pulmonary Hospital (approval no. K19-127Y). The cell classification method proposed in this study is shown in Fig. 1. Patches were cropped from cytological WSIs, and the Aitrox Al model was then used to classify those patches as benign or malignant.

\section{Materials}

In this study, we retrospectively reviewed patients with benign and pulmonary adenocarcinoma pleural effusion cases diagnosed by interventional fine-needle aspiration from March 2018 to January 2020 at Shanghai Pulmonary Hospital. For patients with multiple slides, only one slide with the highest quality was manually selected for downstream analysis. The final constructed dataset consisted of 234 benign and 170 malignant slides, among which $28 \%$ were diagnosed according to cytological morphology alone whereas $72 \%$ were diagnosed according to cytological morphology combined with a matched cell block with IHC. Clinical follow-ups were not included in these cases. All slides were checked by a senior cytopathologist (XFX) and stored in the online digital slide viewer Microscope Image Information System from Shanghai Aitrox Technology Corporation Limited, Shanghai, China. Cytological specimens were prepared by the LCT method and stained with hematoxylin and eosin (H\&E). The LCT is a method used to prepare cytological slides using the characteristics of different types of human cells (cells with larger sizes are rapidly deposited on glass after sedimentation). In pleural effusion cytology, tumor cells are larger than normal cells, which makes them easily recognizable by pathologists. The procedure of cytology specimen preparation was conducted according to the manufacturer's protocol (CytoRich Non-gyn, BD), but with a change involving staining with $\mathrm{H} \& \mathrm{E}$ as used in our daily practice.

Liquid-based cytology can greatly facilitate the digitization of pathology because during the sample preparation, mucus and red blood cells (which often appear in the traditional smear) are removed and other cells are displayed as a thin layer form on the slide. The flat and high-quality slices greatly improve the efficiency of WSI scanning ${ }^{33}$. The WSIs of 234 benign and 170 malignant slides were collected using a digital slide scanner with a $40 \times$ magnification objective and a resolution of $0.25 \mu \mathrm{m} / \mathrm{pixel}$ and saved in SVS format according to the manufacturer's protocol (EasyScan 6, Motic Inc., Xiamen, China). Six slides were excluded due to difficulty in scanning during quality control, including those with out-of-focus blur.

\section{Data distribution}

A consort diagram for data enrollment and allocation is presented in Fig. 2. According to the malignancy distribution of the dataset, 404 WSIs were randomly allocated into training, validation and test sets to train the models, select the best model, and evaluate the performance of the best model ${ }^{34,35}$. Because the sizes of the WSIs were too large to directly input to a neural network, all WSIs were cropped into small patches. There were 266 slides with $1,648,130$ patches in the training set, 78 slides with 451,724 patches in the validation set, and 60 slides with 365,752 patches in the test set. For model construction, 344 slides were split into two groups, namely, the training and validation groups, which optimized the accuracy of the Al model.

\section{Image preparation}

To construct the image dataset for the DCNN, patch images of $512 \times 512$ pixels were first cropped from the original microscopic images without overlapping and then resized to $256 \times 256$ pixels as input for model training. The pathologists reviewed the WSIs, and the number of patches per slide depended on the sample size, with an average of 6104. Before data augmentation, 1,648,130 (754,622 malignant/893,508 benign) and 451,724 (178,392 malignant/273,332 benign) patch images were obtained for the training and validation sets, respectively. As shown in Fig. 3, the patch images included various kinds of cells, and data augmentation (such as random horizontal flipping, random vertical flipping, and color jitter) was introduced to generate more diverse images to improve the generalizability of our model. On average, there were 24,417 patches per slide after data augmentation. Finally, 6,592,520 (3,018,488 malignant/3,574,032 benign) and 1,806,896 (713,568 malignant/1,093,328 benign) augmented patch images were prepared for training and validation, respectively.

\section{DCNN model training}

The weakly supervised WSI classification model relies on multiple instance learning (MIL). The slide-level diagnosis casts the same weak labels on all patches within a specified WSI: on the one hand, if the slide is classified as malignant, at least one patch image contains malignant cells; on the other hand, if the slide is classified as benign, all of its patches must be benign and completely free from malignant cells ${ }^{31}$. The ResNet18 network structure, which was first proposed by Kaiming $\mathrm{He}$, was employed to construct the classification models in this study ${ }^{36}$. A two-step approach was adopted during model training. In the first stage, a classifier was trained with patch images and the corresponding weak labels. The weak label of a single patch image was defined as the label of the WSI within the patch. All patches in the training dataset were inferred by the pretrained classifier, of which the pretrained weights were obtained from pretraining 


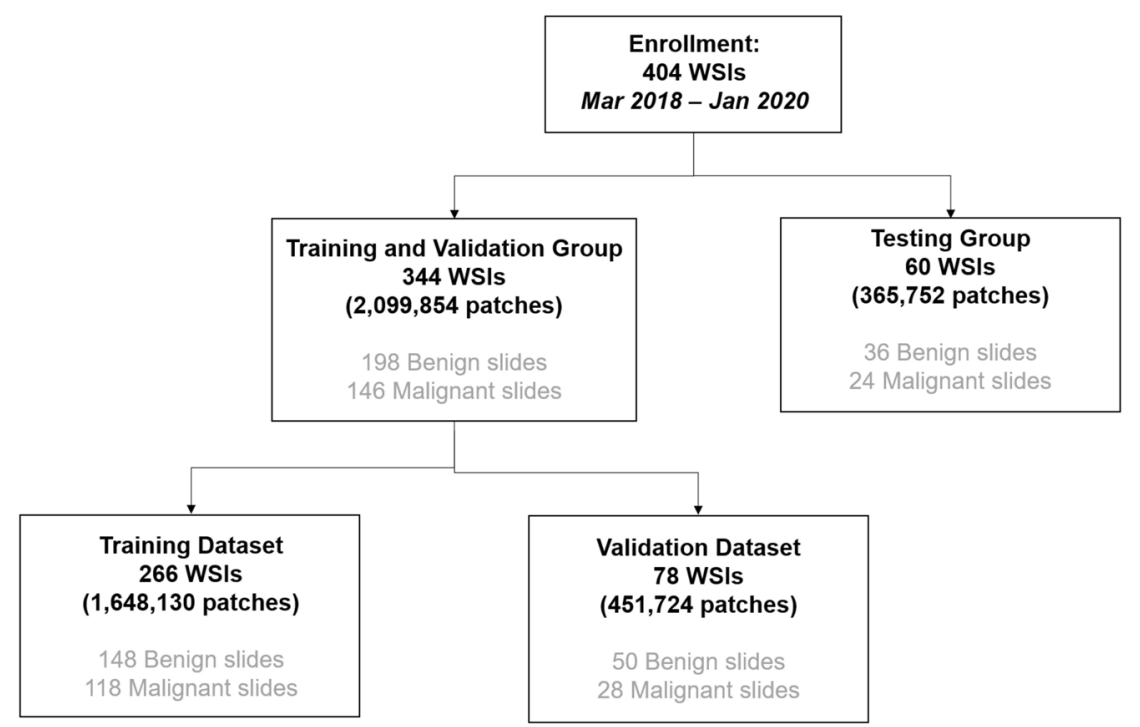

Fig. 2 The consort diagram of the enrolled data from the Shanghai Pulmonary Hospital from March 2018 to January 2020. Enrolled 404 WSIs were randomly allocated into training (266 WSIs), validation (78 WSIs) and testing (60 WSIs) datasets. WSI, whole slide image.

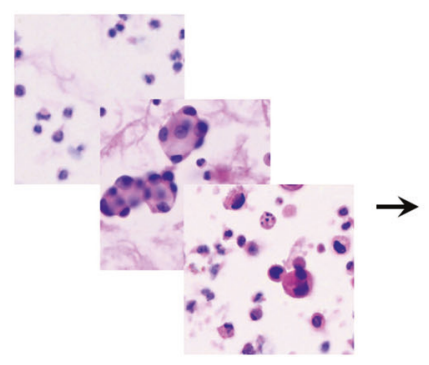

Image patches

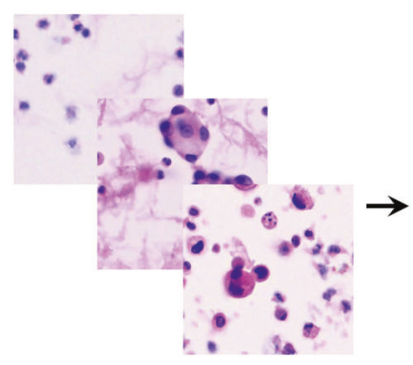

Horizontal flip

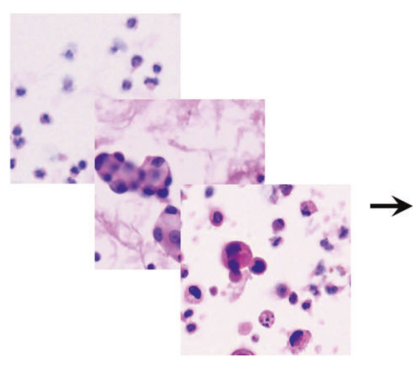

Vertical flip

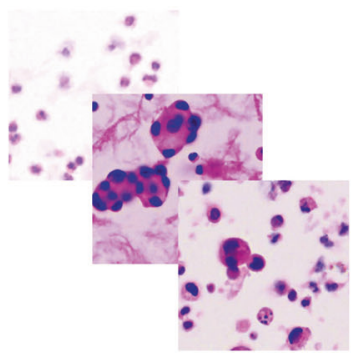

Color jitter

Fig. 3 Generation and augmentation of patch images. Image patches were augmented by horizontal flip, vertical flip and color jitter.

Table 1. Baseline characteristics of the patients in training, validation and testing groups.

\begin{tabular}{|c|c|c|c|c|c|}
\hline Characteristics & Total $(n=404)$ & Training $(n=266)$ & Validation $(n=78)$ & Test $(n=60)$ & $P$ value \\
\hline Age, median (IQR), yr & $66(21-97)$ & $65(21-69)$ & $65(21-91)$ & $65(22-97)$ & 0.1307 \\
\hline Male, No. (\%) & $265(65.27 \%)$ & $174(65.41 \%)$ & $54(69.23 \%)$ & $37(59.68 \%)$ & 0.3837 \\
\hline \multicolumn{6}{|c|}{ Histopathologic \& Clinical final diagnosis } \\
\hline Malignant lesions & $170(41.87 \%)$ & $118(44.36 \%)$ & $28(35.90 \%)$ & $24(38.71 \%)$ & \\
\hline Benign lesions & $234(58.13 \%)$ & $148(55.64 \%)$ & $50(64.10 \%)$ & 36 (61.29\%) & \\
\hline
\end{tabular}

on the ImageNet dataset ${ }^{37}$. The malignant probabilities of the patches inferred within the same slide were sorted from high to low. The top ten patches with the highest probabilities were selected for inclusion in the training set, and each patch obtained a weak label from their common WSI. In the traditional MIL method, $n$ (the number of patches with the highest probabilities) is usually set to 1 . However, since the number of malignant cells in the WSIs of pleural effusion was usually greater than 10, $n$ was set to 10 to obtain more training data in this research, making the model more convergent and preventing overfitting. In addition, to balance the distribution between malignant and benign samples, ten additional patches from each negative WSI were randomly selected for inclusion in the training set. In the second stage, the classifier was updated with the training data obtained from the first stage.

\section{RESULTSTEN}

This study aimed to identify malignant and benign samples. Table 1 shows the baseline characteristics of the patients in all three datasets. The training and test sets achieved a balance in most of the characteristics. The p-values between the training and test sets for age and sex were 0.1307 and 0.3837 , respectively, which are greater than 0.05 and indicate a balanced distribution between the two sets.

The model had an area under the receiver operating characteristic curve (AUC) of 0.9526 with a $95 \%$ confidence interval (Cl) of 0.9019-0.9909 (Fig. 4). If a patch has a malignancy probability greater than 0.50 , it is considered a malignant patch. If a specific WSI has more than 10 malignant patches, it is classified as a malignant slide (Fig. 1).

The case-based classifier's performance was tested on 60 slides and showed $91.67 \%$ accuracy, $87.50 \%$ sensitivity, and $94.44 \%$ specificity. The performance was compared with that of two senior and junior cytopathologists. The cytopathologists annotated the dataset at the WSI level and provided a binary result (malignant or benign) for each WSI. All given outputs were evaluated against the 


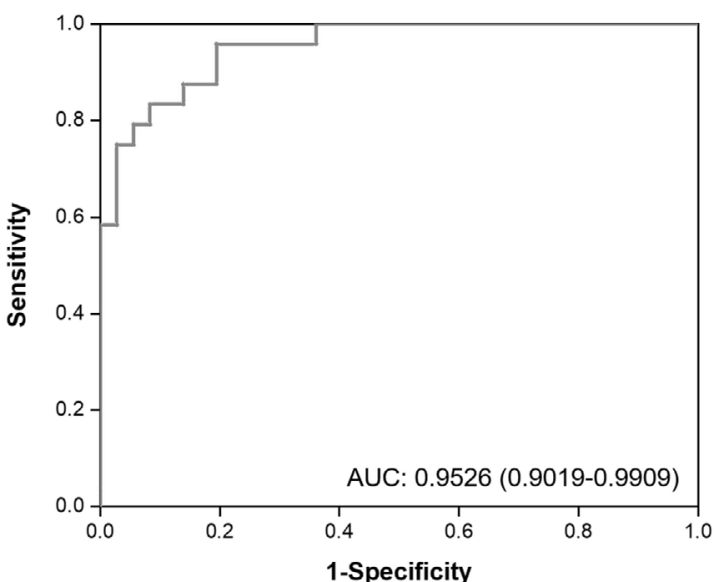

Fig. 4 Receiver operator characteristics (ROC) curve of the Al model with AUC of 0.9526 (95\% Cl: 0.9019-0.9909). AUC denotes the area under the receiver operator characteristics curve.

gold standard (the pathological report result). The accuracy of our Al model was $91.67 \%$, with an AUC of $0.9526(95 \% \mathrm{Cl}$ : 0.9019-0.9909). In comparison, the accuracies of the two senior cytopathologists (P1 and P2; P2 was an expert cytopathologist) and the two junior cytopathologists (P3 and P4) were $96.67 \%$, $100.00 \%, 81.67 \%$, and $85.00 \%$, respectively.

Correlations of the gold standard with the senior cytopathologists, junior cytopathologists, and Aitrox Al model were respectively analyzed. The results showed that the diagnoses by the senior pathologists (P1 and P2, Fig. 5) were most significantly correlated with the gold standard, with $\tau$-values of 0.93 and 1.00. The diagnoses by the junior pathologists (P3 and P4, Fig. 5) showed the lowest correlation with the gold standard, with $\mathrm{t}$-values of 0.64 and 0.71 . The predictions of the Al model were significantly correlated with the gold standard, with a $\tau$-value of 0.83 . The Al predictions also correlated with the diagnoses by the senior and junior cytopathologists, with $\tau$ values ranging from 0.67 and 0.83 . The accuracy of the test set showed that the performance of our Aitrox Al model was between that of the junior and senior cytopathologists (Fig. 5).

Figure 6 shows example patches from malignant and benign WSIs. Fig 6a-d and Fig. 6e-h show correct classifications and misclassifications by our DCNN, respectively.

\section{DISCUSSION}

To the best of our knowledge, this is the first study with the largest dataset that applied weakly supervised Al to cytological pleural effusion image evaluation and compared predictions from Al with diagnoses by cytopathologists at different WSI levels. Previous studies were conducted with a small amount of data at the patch level or single-cell level. For example, Win's experiments on cytological pleural effusion were carried out on $125^{38}$ and $124^{39}$ cytological pleural effusion images. The cytological specimens of 46 patients were collected in Teramoto's study on lung cytological images ${ }^{40}$. In Tosun's study of mesothelial cells in effusion cytology specimens, only 34 patients were enrolled ${ }^{12}$. Compared with these studies, our investigation was carried out with a larger dataset of 404 patients, which indicated that our dataset had broader coverage and higher generalization.

The main purpose of this investigation was to evaluate whether diagnoses made by the weakly supervised Aitrox Al model could reach clinical grade at the WSI level. This was the first time that a weakly supervised deep learning method was introduced to model training to distinguish malignant cells in effusion cytology specimens. To achieve this goal, a deep learning method was developed based on the Resnet 18 structure adopting the MIL

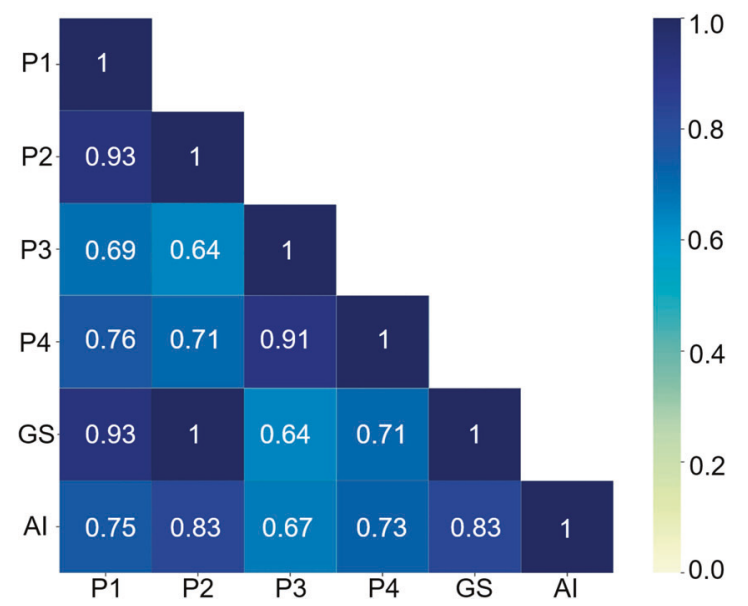

Fig. 5 Heatmap of Kendall correlation $\tau$ value for gold standard, two senior cytopathologists, two junior cytopathologists and DCNN model. GS, Gold standard; P1 and P2, two senior pathologists; P3 and P4, two junior pathologists; Al, the Aitrox Al model based on a DCNN.

approach. Diagnoses were also made by senior cytopathologists, junior cytopathologists and the Aitrox Al model for the same test set comprising 60 WSIs. Kendall's correlation coefficients between the Aitrox Al model and the cytopathologists and gold standard demonstrated that the Aitrox Al model predictions had strong correlations with the diagnoses by all cytopathologists (from 0.67 to 0.83 ) and the gold standard $(0.83)^{41}$. The diagnoses by the senior cytopathologists showed the highest correlation with the gold standard (0.93 and 1.00), while those by the junior cytopathologists showed the lowest correlation with the gold standard (0.64 and 0.71), indicating that the Aitrox Al model has the potential to make clinical-grade decisions during the initial diagnosis.

Among the 60 WSIs in the test set, 5 WSIs were misclassified by our Al model. The Al model performed well in classifying images with straightforward cell morphology and obvious features (Fig. 6a-d). However, the presence of proliferating mesothelial cells that cluster together (Fig. 6e, f) and tumor cells with poor adhesion (Fig. $6 \mathrm{~g}, \mathrm{~h}$ ) may result in misclassification by our DCNN. Of note, the diagnoses made in Fig. $6 e$ and $f$ are also difficult for pathologists in real clinical scenarios, and further evaluation based on cell blocks with IHC by experienced pathologists is necessary.

Cytological diagnosis plays an important role in the rapid diagnosis of lung cancer. Since cytological diagnosis is very subjective and requires experience, it takes a long time to train cytopathologists. Both the severe shortage of cytopathologists and the low accuracy rate of junior cytopathologists limit the applications of cytological diagnosis in China. Our research shows that the Al model has an accuracy rate close to that of senior cytopathologists, which may compensate for the shortage of experienced cytopathologists through its deployment in different hospitals and cities.

In the present study, we explored the performance of a DCNN model in the classification of cancer cells on cytological pleural effusion images at the WSI level. The results showed that the Al model achieved a diagnostic accuracy of $91.67 \%$, which was much better than that of the two junior cytopathologists. The results indicated that Al may assist pathologists in diagnosing cancer cells on cytological pleural effusion images in the future.

This study has some limitations. Our data were collected from a single medical center (Shanghai Pulmonary Hospital), and only lung adenocarcinoma patients were included. While lung adenocarcinoma represents the most common subtype of lung cancer, future studies would benefit from including additional samples of other lung cancer types from multiple national and international centers. 
a

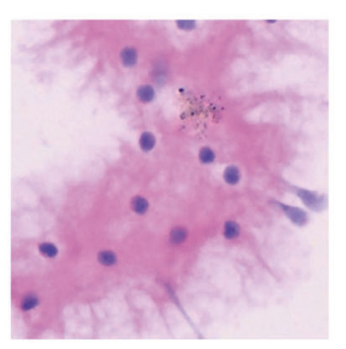

b

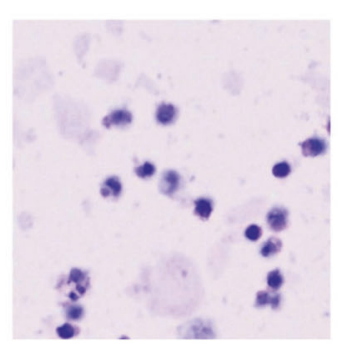

c

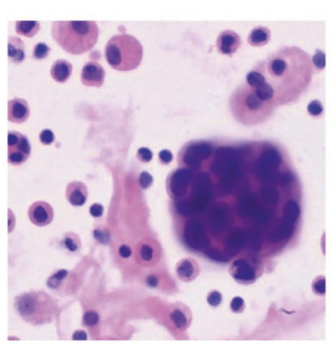

d

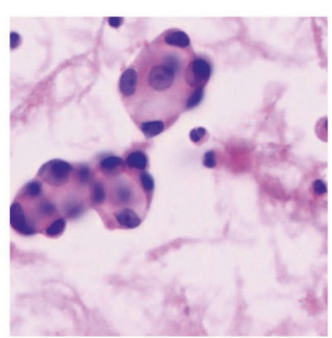

e

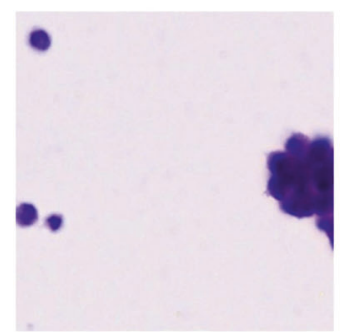

f

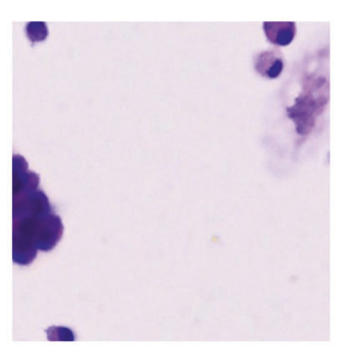

g

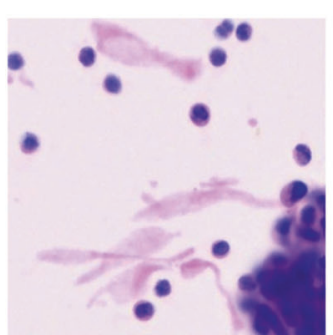

h

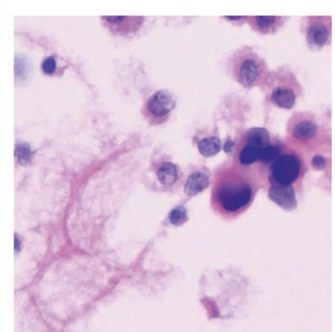

Fig. 6 Different situation of Al performance in pleura effusion cytology diagnosis. a and b show Al made correct diagnosis of benign cells; $\mathbf{c}$ and $\mathbf{d}$ show Al made correct diagnosis of malignant cells; $\mathbf{e}$ and $\mathbf{f}$ show Al misdiagnosed benign mesothelial cells as malignant; $\mathbf{g}$ and $\mathbf{h}$ show Al misdiagnosed malignant cells as benign.

Another potential limitation is the exclusion of any atypical or suspicious diagnoses. A possible direction for future studies to follow up will be developing a DCNN-based method that can not only classify malignant and benign lesions but also output the malignant probability of a WSI to better assist pathologists in diagnosing atypical or suspicious cases.

\section{DATA AVAILABILITY}

The datasets used and/or analyzed during the current study are available from the corresponding author on reasonable request.

\section{REFERENCES}

1. Jiang, T. et al. The changing diagnostic pathway for lung cancer patients in Shanghai, China. Eur. J. Cancer 84, 168-172 (2017).

2. Shlomi, D., Ben-Avi, R., Balmor, G. R., Onn, A. \& Peled, N. Screening for lung cancer: time for large-scale screening by chest computed tomography. Eur. Respir. J. 44, 217-238 (2014)

3. Bhandari, S., Pham, D., Pinkston, C., Oechsli, M. \& Kloecker, G. Timing of treatment in small-cell lung cancer. Med. Oncol. 36, 47 (2019).

4. Sato, T. et al. Prognostic understanding at diagnosis and associated factors in patients with advanced lung cancer and their caregivers. Oncologist 23 1218-1229 (2018).

5. Sermanet, P. et al. Overfeat: Integrated recognition, localization and detection using convolutional networks. arXiv 1312, 6229 (2014).

6. Zeiler M. D., Fergus R. Visualizing and understanding convolutional neural networks. In ECCV, 818-833 (2014).

7. Cicek, T. et al. Adequacy of EBUS-TBNA specimen for mutation analysis of lung cancer. Clin. Respir. J. 13, 92-97 (2019).

8. Sundling, K. E. \& Cibas, E. S. Ancillary studies in pleural, pericardial, and peritoneal effusion cytology. Cancer Cytopathol. 126, 590-598 (2018).

9. Baburaj, G. et al. Liquid biopsy approaches for pleural effusion in lung cancer patients. Mol. Biol. Rep. 47, 8179-8187 (2020).

10. Akamatsu, $\mathrm{H}$. et al. Multiplexed molecular profiling of lung cancer using pleural effusion. J. Thorac. Oncol. 9, 1048-1052 (2014).

11. Ruan, X. et al. Multiplexed molecular profiling of lung cancer with malignant pleural effusion using next generation sequencing in Chinese patients. Oncol. Lett. 19, 3495-3505 (2020).

12. Tosun, A. B., Yergiyev, O., Kolouri, S., Silverman, J. F. \& Rohde, G. K. Detection of malignant mesothelioma using nuclear structure of mesothelial cells in effusion cytology specimens. Cytom A 87, 326-333 (2015).

13. Sakr, L. et al. Cytology-based treatment decision in primary lung cancer: is it accurate enough? Lung Cancer 75, 293-299 (2012)
14. Edwards, S. L. et al. Preoperative histological classification of primary lung cancer: accuracy of diagnosis and use of the non-small cell category. J. Clin. Pathol. $\mathbf{5 3}$ 537-540 (2000)

15. Matsuda, M. et al. Bronchial brushing and bronchial biopsy comparison of diagnostic accuracy and cell typing reliability in lung cancer. Thorax 41, 475-478 (1986).

16. DiBonito, L. et al. Cytological typing of primary lung cancer study of 100 cases with autopsy confirmation. Diagn. Cytopathol. 7, 7-10 (1991).

17. Krizhevsky, A., Sutskever, I. \& Hinton, G. Imagenet classification with deep convolutional neural networks. Commun. ACM 60, 84-90 (2017).

18. Cun, Y. L. et al. Backpropagation applied to handwritten zip code recognition Neural Comput. 1, 541-551 (1989).

19. Muhammad, K. K. N., Parwani, A. V. \& Gurcan, M. N. Digital pathology and artificial intelligence. Lancet Oncol. 20, e253-e261 (2019).

20. Miller, D. D. \& Brown, E. W. Artificial intelligence in medical practice: the question to the answer? Am. J. Med. 131, 129-133 (2018).

21. Pallua, J. D., Brunner, A., Zelger, B., Schirmer, M. \& Haybaeck, J. The future of pathology is digital. Pathol. Res. Pract. 216, 153040 (2020).

22. Bera, K., Schalper, K. A., Rimm, D. L., Velcheti, V. \& Madabhushi, A. Artificial intelligence in digital pathology-new tools for diagnosis and precision oncology. Nat. Rev. Clin. Oncol. 16, 703-715 (2019).

23. Antonio, V. A. A. et al. Classification of lung adenocarcinoma transcriptome subtypes from pathological images using deep convolutional networks. Int. J. Comput. Assist. Radio Surg. 13, 1905-1913 (2018).

24. Teramoto, A. et al. Deep learning approach to classification of lung cytological images: Two-step training using actual and synthesized images by progressive growing of generative adversarial networks. PLoS One 15, e0229951 (2020).

25. Wei, J. et al. Pathologist-level classification of histologic patterns on resected lung adenocarcinoma slides with deep neural networks. Sci. Rep. 9, 3358 (2019).

26. Kriegeskorte, N. \& Golan, T. Neural network models and deep learning. Curr. Biol. 29, R231-R236 (2019).

27. Lepus, C. M. \& Vivero, M. Updates in effusion cytology. Surg. Pathol. Clin. 11, 523-544 (2018)

28. Humphries, M. P. et al. Automated tumour recognition and digital pathology scoring unravels new role for PD-L1 in predicting good outcome in ER-/HER2+ breast cancer. J. Oncol. 2018, 2937012 (2018).

29. Sha, L. et al. Multi-field-of-view deep learning model predicts non-small cell lung cancer Programmed Death-Ligand 1 status from whole-slide Hematoxylin and Eosin images. J. Pathol. Inform. 10, 24 (2019).

30. Kanavati, F. et al. Weakly-supervised learning for lung carcinoma classification using deep learning. Sci. Rep. 10, 9297 (2020)

31. Campanella, G. et al. Clinical-grade computational pathology using weakly supervised deep learning on whole slide images. Nat. Med. 25, 1301-1309 (2019).

32. Girum, K. B., Crehange, G., Hussain, R. \& Lalande, A. Fast interactive medical image segmentation with weakly supervised deep learning method. Int. J. Comput. Assist. Radio Surg. 15, 1437-1444 (2020). 
33. Simone, L. V. E. et al. Constant quest for quality digital cytopathology. J. Pathol. Inf 9, 13 (2018).

34. Coudray, N. et al. Classification and mutation prediction from non-small cell lung cancer histopathology images using deep learning. Nat. Med. 24, 1559-1567 (2018).

35. Khalid, A. et al. Geospatial immune variability illuminates differential evolution of lung adenocarcinoma. Nat. Med. 26, 1054-1062 (2020).

36. He K., Zhang X., Ren S., Sun J. Deep residual learning for image recognition. In CVPR, 770-778 (2016).

37. Deng J., et al ImageNet: a large-scale hierarchical image database. In CVPR, 248-255 (2009).

38. Win, K. Y. et al. Computer aided diagnosis system for detection of cancer cells on cytological pleural effusion images. Biomed. Res. Int. 2018, 6456724 (2018).

39. Win, K., Choomchuay, S., Hamamoto, K. \& Raveesunthornkiat, M. Detection and classification of overlapping cell nuclei in cytology effusion images using a double-strategy random forest. Appl. Sci. 8, 1608 (2018).

40. Teramoto, A. et al. Automated classification of benign and malignant cells from lung cytological images using deep convolutional neural network. Inform. Med. Unlocked 16, 100205 (2019).

41. Akoglu, H. User's guide to correlation coefficients. Turk. J. Emerg. Med. 18, 91-93 (2018).

\section{AUTHOR CONTRIBUTIONS}

W.C.Y. performed study concept and design; X.F.X wrote the manuscript; Yue Yu, Q.Y. Y. made Algorithm implementation; Chi-Cheng Fu analysis and interpretation of data, and statistical analysis; Q.F. provided technical support; L.P.Z., L.K.H., W.C.Y. performed development of methodology and writing, review and revision of the paper; All authors read and approved the final paper.

\section{FUNDING}

This work was supported by projects from The Shanghai Municipal Health Commission (20194Y0228), Shanghai Hospital Development Center Foundation (SHDC2020CR3047B), Clinical Research Foundation of Shanghai Pulmonary Hospital (fk1937), Shanghai Science and Technology Commission (18411962900).

\section{COMPETING INTERESTS}

Authors have no conflict of interest to declare. I would like to declare on behalf of my co-authors that the work described was original research that has not been published previously, and not under consideration for publication elsewhere, in whole or in part. All the authors listed have approved the manuscript that is enclosed.

\section{ETHICS APPROVAL}

This study was approved by the Ethics Committee of Shanghai Pulmonary Hospital (approval no. K19-127Y).

\section{ADDITIONAL INFORMATION}

Correspondence and requests for materials should be addressed to Chunyan $\mathrm{Wu}$.

Reprints and permission information is available at http://www.nature.com/ reprints

Publisher's note Springer Nature remains neutral with regard to jurisdictional claims in published maps and institutional affiliations.

Open Access This article is licensed under a Creative Commons Attribution 4.0 International License, which permits use, sharing, adaptation, distribution and reproduction in any medium or format, as long as you give appropriate credit to the original author(s) and the source, provide a link to the Creative Commons license, and indicate if changes were made. The images or other third party material in this article are included in the article's Creative Commons license, unless indicated otherwise in a credit line to the material. If material is not included in the article's Creative Commons license and your intended use is not permitted by statutory regulation or exceeds the permitted use, you will need to obtain permission directly from the copyright holder. To view a copy of this license, visit http://creativecommons. org/licenses/by/4.0/.

(c) The Author(s) 2022 